\title{
Lower level of complement component C3 and C3a in the plasma means poor outcome in the patients with hepatitis $B$ virus related acute-on-chronic liver failure
}

\section{Qian Li}

Department of Infectious diseases, Huashan Hospital, Fudan University

\section{Qing Lu}

Department of Infectious disease, Huashan Hospital, Fudan University

\section{Meng-Qi Zhu}

Department of Infectious diseases, Huashan Hospital, Fudan University

\section{Chong Huang}

Department of infectious disease, Huashan Hospital, Fudan University

\section{Kang-Kang Yu}

Department of infectious diseases, Huashan Hospital, Fudan University

\section{Yu-Xian Huang}

Department of infectious diseases, Huashan Hospital, Fudan University

\section{Xu Zhao}

Institute of Antibiotics, Huashan Hospital, Fudan University

\section{Xing-Guang Luo}

Department of Genetics, Yale University

Jian-Ming Zheng ( $\nabla$ zhengjianming@fudan.edu.cn )

Fudan University https://orcid.org/0000-0003-1471-9078

\section{Research article}

Keywords: Complement, hepatitis B virus, acute-on-chronic liver failure

Posted Date: January 17th, 2020

DOI: https://doi.org/10.21203/rs.2.21119/v1

License: (c) (1) This work is licensed under a Creative Commons Attribution 4.0 International License. Read Full License 
Version of Record: A version of this preprint was published at BMC Gastroenterology on April 15th, 2020. See the published version at https://doi.org/10.1186/s12876-020-01258-3. 


\section{Abstract}

Background The purpose of this study was to investigate whether or not the complement system is activated systemically activated and to specify the clinical and prognostic implications of its components during hepatitis $B$ virus related acute-on-chronic liver failure (HBV-ACLF).

Methods Blood samples from twenty-seven patients diagnosed with HBV-ACLF, twenty-five patients diagnosed with chronic hepatitis $B$ but without liver failure, and nine health volunteers were enrolled in this survey. Plasma complement components were measured with Enzyme-linked immunosorbent assay. Correlative analysis were assessed between the levels of complement components and the liver failure related index.

Results The concentrations of $\mathrm{C} 1 \mathrm{q}, \mathrm{C} 3, \mathrm{C} 3 \mathrm{a}, \mathrm{C} 4, \mathrm{C} 4 \mathrm{a}$ and $\mathrm{s} C 5 \mathrm{~b}-9$ were significantly higher in the control group than those in the HBV-ACLF group (3.5, 2.4, 2.1, 1.4, 1.3 and 6.0 fold, respectively). However, there was no statistical significance of the differences in the plasma concentrations of mannose binding lectin and factor $B$ between the HBV-ACLF and control groups. The levels of C3 and C3a were inversely correlated with MELDs $(P<0.05)$ or CLIF-C OFs $(P<0.05)$.

Conclusions Our data demonstrated that the plasma levels of C3 and C3a might be potential novel biomarkers to predict the outcome of HBV-ACLF.

\section{Introduction}

The acute-on-chronic liver failure (ACLF) is characterized by hepatic failure and/or by multi-organ failure and high 28-day mortality. ACLF is a severe disease that many patients need liver transplantation as a treatment choice. Hepatitis B virus (HBV) is still the main reason of ACLF in the Asian region[1].

The pathophysiology of the ACLF has not been fully explored. Several reports have observed that systemic inflammation is a hallmark of ACLF[2]. ACLF patients show higher plasma levels of proinflammatory cytokines [such as the interleukin- (IL-) 6 and tumor necrosis factor- (TNF-) a], white blood cell count and $C$ reactive protein (CRP) than in healthy individuals $[3,4]$. The inflammatory inducers could be both exogenous and endogenous factors, including the microbial and nonmicrobial factors, such as the context of the tissue damage or injury. The complement system plays an important role in both host innate immune defense and inflammatory progress of human diseases[5-10]. The activation of the complement system in response to invading pathogens is initiated through the classical, the alternative and the lectin pathways. The initiator of the three activation pathway are C1q, Factor B (FB) and Mannose binding lectin (MBL), respectively. The complement activation results in the $C 3$ cleavage, the anaphylatoxins $\mathrm{C} 3 \mathrm{a}$ and $\mathrm{C} 5 \mathrm{a}$ release, and the formation of a membrane attack complex (sC5b-9) to lyse the target cells. The liver is the major site for complement synthesis[11]. The complement system enhanced susceptibility of steatotic livers to ischemia and reperfusion injury. The complement component 3 (C3) deficiency, the inhibition of complement receptor 2-complement receptor 1-related protein y (an inhibitor of C3 activation) could provide the protection from hepatic ischemia and 
reperfusion injury in mice[12,13]. In addition, the activation of the complement system plays a key role in hepatic inflammation and progression of injury during the pathogenesis of acetaminophen-induced hepatotoxicity[14]. However, whether or not the complement system plays the pivotal role in the pathogenesis of HBV-related ACLF (HBV-ACLF) is still unknown[15]. To our knowledge, only one study reported serum levels of the complement 3 and 4 ( $\mathrm{C} 3$ and $\mathrm{C} 4)$, and the complement function (CH50) in HBV-ACLF patients and the result suggested C3 level can be a prognostic marker for the mortality of the disease[15].

In nonalcoholic fatty liver disease, there is a widespread activation of the complement system which is related to the disease severity[16]. Whether the complement system had a relationship with the prognosis in HBV-ACLF or not remains largely unknown. The model for end-stage liver disease (MELD) is a conventional scoring system used as a prognostic tool devised for the end-stage liver disease and the utility of transplantation[17]. However, our previous study revealed that chronic liver failure (CLIF) consortium organ failure score (CLIF-C OF) enables more accurate prediction of short-term mortality in patients with HBV-ACLF than MELD, CLIF sequential organ failure assessment score (CLIF SOFA), and CLIF consortium ACLF score (CLIF-C ACLF)[18]. Therefore, the correlative analysis between the complement components levels and the liver failure related index, including prognostic scoring system, such as MELD and CLIF-C OF, were assessed in this study.

The purpose of this study was to investigate whether or not the complement system is systemically activated as well as its relationship with the prognosis in HBV-ACLF. The levels of the complement components plasma samples from HBV-ACLF patients were determined and compared with those in the control group. Correlative analyses between the complement components levels and the liver failure related index were also examined.

\section{Materials And Methods}

Patients selection, clinical sample collection and processing

About amongst the 27 consecutive samples with HBV-ACLF and 25 samples with chronic hepatitis B as a discharge diagnosis were screened. They were treated at the Department of Infectious Diseases, Huashan Hospital, Fudan University (Shanghai, China) from February 2014 to June 2017. Exclusion criteria were: 1) patients aged less than 14 years; 2) patients co-infected with human immunodeficiency virus; 3 ) patients with the coexistence of liver injury caused by any other etiologies including hepatitis $C$ or $D$ virus infection, drug intake, alcohol consumption and autoimmune hepatitis, etc. 4) pregnancy and lactation (Table 1).

The diagnosis of ACLF was based on the criteria formalized by the acute-on-chronic liver failure consensus recommendations of the Asian Pacific Association for the Study of the Liver (APASL) 2014[1]. Acute liver failure is generally defined as the development of hepatic encephalopathy within 4 weeks of onset of jaundice[1]. Since the basic premise in ACLF is to identify patients with chronic liver disease or cirrhosis presenting as acute liver failure, the time frame for liver failure was kept as 4 weeks. Acute-on- 
chronic liver failure is defined as the coagulation abnormality usually with an international normalized ratio (INR) $\geq 1.5$ and total bilirubin $\geq 10 \mathrm{mg} / \mathrm{dl}$ in this study. The diagnosis of chronic hepatitis $\mathrm{B}$ (CHB) was defined as the presence of HBsAg in serum for more than 6 months, high levels of HBV DNA, and by persistently elevated serum alanine aminotransferase without the other reasons[19]. The patients in chronic hepatitis B group were without liver failure. The control group was made up of nine healthcare volunteers. Venous blood was collected when the patients were one day after admission, were centrifuged and single use aliquots of these samples were stored at $-80^{\circ} \mathrm{C}$. The study was performed in accordance with the 1964 Declaration of Helsinki and was approved by the Ethical Committee of Huashan Hospital, Fudan University. If the participants were less than 18 years old, the written consent was instead obtained from the parents/legal guardians of these participants on their behalf.

Clinical characteristics and biochemical parameters

Clinical characteristics including all the components of various prognostic scores and blood parameters (routine blood tests, coagulation function tests, serum electrolyte levels, liver and renal function tests, and arterial blood gas analysis) were analyzed.

\section{Prognostic scores}

The CLIF-OF score has been shown to accurately predict short-term mortality in patients with HBV-ACLF in our previous study[18]. We used the published formula to compute CLIF-C OFs and MELDs in our cohort[2, 17, 20]. The CLIF-C OFs (range 6-18) at diagnosis was defined by the presence of hepatic, renal, cerebral, coagulatory, circulatory and respiratory failure[20]. MELD score was calculated as following: $9.6 \times \ln [$ creatinine $(\mathrm{mg} / \mathrm{dL})]+3.8 \times \ln [$ bilirubin $(\mathrm{mg} / \mathrm{dL})]+11.2 \times \ln (\mathrm{INR})+6.4 \times($ etiology: 0 if cholestatic or alcoholic, 1 otherwise)[17].

\section{ELISA}

Human Complement C1q, FB, MBL, C3, C3a, C4, C4a and sC5b-9 were measured with commercial ELISA kits (Cusabio Biotech, Wuhan, P.R. China; BD biosciences, San Diego, USA; Abcam, Cambridge, UK, QuidelSan Diego, USA; R\&D Systems, Minneapolis, USA)[7]. Experiment protocols were followed according to the manufacturer's instructions.

Western blot 
For comparison of C3 activation in plasma samples, about $2 \mu \mathrm{l}$ of plasma samples were loaded on an SDS-PAGE gel. Samples were transferred to polyvinylidene difluoride membranes. The membranes were blocked and then incubated with mouse anti-human C3/C3b/iC3b/C3dg monoclonal antibody (1:1000, Cedarlane, Ontario, Canada) in TBST (Tris-buffered saline supplemented with Tween 20). Blots were then washed and incubated with goat anti-mouse IgG (1:15000, LI-COR Bioscience, Nebraska, USA) diluted in TBST. The membranes were developed with an Odyssey system (Li-COR Bioscience) according to the manufacturer's protocol.

Statistical analysis

Statistical analyses were performed with the Graphpad 5.0 (Graphpad Software, San Diego, CA, USA). Variables were expressed as mean \pm standard deviation unless otherwise specified. Differences in the parameters were compared by using the non-parametric Mann-Whitney U-test and the relationship between two variables was assessed by Pearson correlation or Spearman's rank correlation, respectively. A two-tailed $P$ value of $<0.05$ was considered statistically significant.

\section{Results}

\section{Baseline Characteristics}

All patients were Chinese. Table 1 shows the baseline characteristics at enrollment in the study. There were no significant differences in gender, age and creatinine in all groups. Nevertheless, the levels of alanine aminotransferase (ALT), total bilirubin, INR, AFP and MELD scores were significantly higher in ACLF group than that in the other group.

Complement components in plasma samples

The plasma concentrations of C1q were markedly reduced in HBV-ACLF than those in CHB group or in control group, but not the MBL and FB

We first examined the concentrations of the complement classical pathway component (C1q), the lectin pathway (MBL) and the alternative pathway (FB) in plasma samples from HBV-ACLF, CHB and control group. The concentrations of $\mathrm{C} 1 \mathrm{q}$ were significantly lower in HBV-ACLF group than that in CHB group or in control group(Fig. 1A). The mean concentration of C1q was $177001 \mathrm{ng} / \mathrm{ml}$ in the HBV-ACLF group, but only $114640 \mathrm{ng} / \mathrm{ml}$ in CHB group and $50509 \mathrm{ng} / \mathrm{ml}$ in control group. However, There were no statistical significances of the plasma concentrations of MBL and FB in all groups(Fig. 1B-C), suggesting that the classical pathway plays the more important role in the inflammatory activities of HBV-ACLF. (Table 1) 
The concentrations of complement components $\mathrm{C} 3, \mathrm{C} 4$, their degradation products and sC5b-9 in all groups

Complement C3 and its degradation product C3a were significantly higher in the control group than that in the HBV-ACLF group(Fig. 1D-E). The C3 is $15653 \mu \mathrm{g} / \mathrm{ml}$ in the control group, $8916 \mu \mathrm{g} / \mathrm{ml}$ in the CHB group and $6568 \mu \mathrm{g} / \mathrm{ml}$ in the HBV-ACLF group. C3 and its degradation product C3a were significantly higher in the control group than that in the HBV-ACLF group. The C4 is $1530 \mu \mathrm{g} / \mathrm{ml}$ in the control group, $1487 \mu \mathrm{g} / \mathrm{ml}$ in the CHB group and $1074 \mu \mathrm{g} / \mathrm{ml}$ in the HBV-ACLF group(Fig. 1F-H). The C3 and C3a level in ACLF survival group was higher than that in ACLF non-survival group, but without statistical difference. The concentrations of C1q, C3, C3a, C4, C4a and sC5b-9 were significantly higher in the control group than those in the HBV-ACLF group (3.5, 2.4, 2.1, 1.4, 1.3 and 6.0 fold, respectively). (Table 2)

The relationship between complement components and clinical parameter

The concentrations of $\mathrm{C} 1 \mathrm{q}, \mathrm{MBL}$ and $\mathrm{FB}$ were not correlated with the prognosis score

There are no statistical significance of the plasma concentrations of $\mathrm{C} 1 \mathrm{q}, \mathrm{MBL}$ and FB between the ACLF survival group and ACLF non-survival group. None of them were correlated with ALT, total bilirubin, albumin and HBV DNA. Only the C1q were observed to be positively correlated with AFP $(P<0.05)$. (Fig. 2A)

The correlation of $\mathrm{C} 3, \mathrm{C} 4$ and their degradation products levels with clinical parameter and prognosis score

C3 were observed to be positively correlated with AFP $(P<0.05)$. C3a was observed to be positively correlated with $\mathrm{C} 3, \mathrm{r}=4675$, but $\mathrm{P}=0.0504$. $\mathrm{C} 3 \mathrm{a}$ and $\mathrm{C} 4 \mathrm{a}$ were observed to be negatively correlated with creatinine $(P<0.05)$. $C 4$ was observed to be negatively correlated with INR $(P<0.05)$. But none of them were correlated with ALT, total bilirubin, albumin and HBV DNA (Fig. 2B-E). C3, C3a and C4a were observed to be negatively correlated with MELDs $(P<0.05)$ (Fig. 3A-C). C3, C3a and C4 were observed to be negatively correlated with CLIF-C OFs $(P<0.05)$.

C3 activation in plasma in ACLF group

The complement system activation is mediated through the classical , the alternative and the lectin pathways. Each pathway converges at the $\mathrm{C} 3$ convertase level resulting in $\mathrm{C} 3$ cleavage. To further assess whether or not the C3 activation is involved in ACLF group, levels of C3 breakdown products in plasma samples were evaluated by Western blot. According to C3 level, we adjusted sample's volume to ensure the samples' C3 concentration from two groups is the same for comparability. We repeated western blot 
test at least three times. Under the same conditions, C3 cleavage fragments iC3b were detected in both groups. Besides, and the level of iC3b was more in ACLF group than the one in CHB group. C3 cleavage fragments iC3b was detected in plasma from both ACLF group and CHB group. Prominent iC3b band was found in plasma samples in ACLF patients. (Fig. 4)

\section{Discussion}

The immune responses and the inflammatory cascades are important pathogenic mechanisms during ACLF. Though most of the plasma complement factors are derived from the liver, the role of the complement system in liver diseases is not completely understood, and few studies were reported for HBV-ACLF[4].

In response to acute injury, Kupffer cells are activated through complement C3 and C5 receptor signaling pathways[4]. There are, however, no reports assessing how complement is activated in HBV-ACLF patients. In this study, we systematically determined the complement activation, and we firstly showed decreased concentrations of $\mathrm{C} 1 \mathrm{q}, \mathrm{C} 3 \mathrm{a}, \mathrm{C} 4, \mathrm{C} 4 \mathrm{a}$ and sC5b-9 in HBV-ACLF patients compared to that in the control group. C3 and C4 levels were significantly lower in HBV-ACLF patients, which is similar to a previous study[15]. Some studies have shown that the MBL level in acute liver failure patients was $40 \%$ lower compared with healthy controls, and spontaneous acute liver failure survivors had higher levels of MBL at day 1 and lower levels of L-ficolin by day 3 compared with patients who dead or transplanted patients [21]. Despite that, we couldn't see any difference of FB and MBL levels between the HBV-ACLF patients and the control group. This phenomenon may due to the different reason of the disease, but further study need to be done to clarify the mechanism.

We investigated the correlation between complement components and prognostic scores in HBV-ACLF patients, and found that C3 and C3a levels were negatively correlated with MELDs and CLIF-C OFs. Therefore, the synergistic protective activity of both C3 and C3a may further improve their diagnostic value in predicting disease progression. The complement components might be a potential biomarker to predict disease outcome in HBV-ACLF patients. Interestingly, the higher level of C3 and C3a means the higher survival rates in HBV-ACLF patients in CLIF-C OF score model, which is a similar to the result of a previous study about C3 level in HBV-ACLF patients[15].

The complement has been described as a double-edged sword since activation of the complement system significantly contributes to the pathogenesis of various acute and chronic inflammatory diseases, but it also plays a significant role in the resolution of inflammation and tissue repair[22]. C3a has been reported its anti-inflammatory and regenerative effects for normal liver recovery after toxic injury[23]. Experimental evidence has demonstrated that complement anaphylatoxins $\mathrm{C} 3 \mathrm{a}$ and $\mathrm{C} 5 \mathrm{a}$ are required for the survival of liver cells during regeneration[24-27]. Further studies, carried out to explore the mechanisms responsible for the protective role of C3a during HBV-ACLF are warranted.

We found that C3a might be positively correlated with $C 3(r=4675$, but $P=0.0504)$. It suggests that if HBV-ACLF patients have more functional liver cells, more C3 can be synthesized and more complement 
effectors C3a can be produced during complement activation.

Hepatorenal syndrome usually indicates poor prognosis of liver failure. The main clinical signs of the hepatorenal syndrome are the progressive oliguria and anuria and the rising levels of the urea nitrogen and blood creatinine. Our study has shown that C3a and C4a were negatively correlated with creatinine. INR is another important biochemical marker, and it is included in MELD and CLIF OF score systems. Results have shown that the levels of C4 were negatively correlated with INR. AFP is not only a marker of hepatocellular carcinoma, but also a biomarker of liver regeneration. We found out that $\mathrm{C} 1 \mathrm{q}$ and $\mathrm{C} 3$ were positively correlated with AFP, which means that the patients with higher C1q and C3 might recover better.

The C3 breakdown products iC3b was more in ACLF group than the one in CHB group, which as observed by Western blot. The increase of iC3b suggests that complement system is more activated in the ACLF group than the CHB group. This may be an important mechanism for ACLF. But C3a has been reported its anti-inflammatory and regenerative effects for normal liver recovery after toxic injury. The exact role of complement system in ACLF is unknown. Further study is needed to clarify the mechanism.

\section{Conclusions}

In conclusion, our data suggest that the complement system is activated in patients with HBV-ACLF. Increased levels of C3 and C3a seem to be associated with better prognosis during HBV-ACLF, and can be potential novel biomarkers to predict disease outcome. The role of the complement system activation in host defense and neuro-inflammation in HBV-ACLF patients remains to be fully explored. Highlighting which components of the complement system are important in both protective and inflammatory roles in HBV-ACLF patients could drive development of ACLF-specific immunotherapies.

\section{Abbreviations}

ACLF: acute-on-chronic liver failure; ALT: alanine aminotransferase; APASL: Asian Pacific Association for the Study of the Liver; CLIF: chronic liver failure; CLIF-C ACLF: CLIF consortium ACLF score; CLIF-C OF: CLIF consortium organ failure score; CLIF SOFA: CLIF sequential organ failure assessment score; CRP: $C$ reactive protein; C: complement; FB: Factor B; HBeAg: hepatitis B virus e antigen; HBV: Hepatitis B virus; HBV-ACLF: HBV-related ACLF; INR: international normalized ratio; IL: Interleukin; MBL: Mannose binding lectin; MELD: The model for end-stage liver disease; TNF-a: tumor necrosis factor-a

\section{Declarations}

Acknowledgements

This work was supported by Natural Science Foundation Project of Shanghai (No.19ZR1407800), National Natural Science Foundation of China (award number 81371821 and 81670560) and Health and Family Planning Commission of Shanghai (award number 201440578). 
We thank Dr. Hua Hua Tong from the Ohio State University for her helpful suggestions and criticism.

\section{Authors' contributions}

QL1, QL2 and XZ contributed to the study idea; MZ contributed to ELISA test; $\mathrm{CH}, \mathrm{KY}, \mathrm{YH}, \mathrm{XQL}$ and QL2 contributed to clinical data collection; JZ contributed to samples detection and manuscript writing. QL1 is corresponding to Qian Li and QL2 is corresponding to Qing Lu. All authors read and approved the final manuscript.

Consent for publication

Not Applicable.

Availability of data and materials

Data supporting our findings is contained within the manuscript. Data is available from the corresponding author upon request. Identifying/confidential patient data however will not be shared.

Competing interests

The authors declare that they have no competing interests.

\section{Ethical approval}

The study was performed in accordance with the 1964 Declaration of Helsinki and was approved by the Ethical Committee of Huashan Hospital, Fudan University. If the participants were less than 18 years old, the written consent was instead obtained from the parents/legal guardians of these participants on their behalf.

Informed consent

Clinical samples were collected from patients after written informed consent was obtained. 
Author details

${ }^{1}$ Department of Infectious Diseases, Huashan Hospital, Fudan University, Shanghai 200040, China.

${ }^{2}$ Institute of Antibiotics, Huashan Hospital, Fudan University, Shanghai 200040, China. ${ }^{3}$ Georgia State University, Georgia, USA. ${ }^{4}$ Department of Genetics, Yale University School of Medicine, 333 Cedar Street, New Haven, CT 06510, USA.

\section{References}

1. Sarin SK, Kedarisetty CK, Abbas Z, Amarapurkar D, Bihari C, Chan AC, et al. Acute-on-chronic liver failure consensus recommendations of the Asian Pacific Association for the Study of the Liver (APASL) 2014. Hepatol Int. 2014; 8:453-71.

2. Moreau R, Jalan R, Gines P, Pavesi M, Angeli P, Cordoba J, et al. Acute-on-chronic liver failure is a distinct syndrome that develops in patients with acute decompensation of cirrhosis. Gastroenterology. 2013; 144:1426-37.

3. Desmots F, Rissel M, Gilot D, Lagadic-Gossmann D, Morel F, Guguen-Guillouzo C, et al. Proinflammatory cytokines tumor necrosis factor alpha and interleukin- 6 and survival factor epidermal growth factor positively regulate the murine GSTA4 enzyme in hepatocytes. J Biol Chem. 2002; 277:17892-900.

4. Sarin SK, Choudhury A. Acute-on-chronic liver failure: terminology, mechanisms and management. Nat Rev Gastroenterol Hepatol. 2016; 13:131-49.

5. Li Q, Li YX, Stahl GL, Thurman JM, He Y, Tong HH. Essential role of factor B of the alternative complement pathway in complement activation and opsonophagocytosis during acute pneumococcal otitis media in mice. Infect Immun. 2011; 79:2578-85.

6. Li Q, Li YX, Douthitt K, Stahl GL, Thurman JM, Tong HH. Role of the alternative and classical complement activation pathway in complement mediated killing against Streptococcus pneumoniae colony opacity variants during acute pneumococcal otitis media in mice. Microbes Infect. 2012; 14:1308-18.

7. Shen L, Zheng J, Wang Y, Zhu M, Zhu H, Cheng Q, et al. Increased activity of the complement system in cerebrospinal fluid of the patients with Non-HIV Cryptococcal meningitis. BMC Infect Dis. 2017; $17: 7$.

8. Ricklin D, Hajishengallis G, Yang K, Lambris JD. Complement: a key system for immune surveillance and homeostasis. Nat Immunol. 2010; 11:785-97.

9. Tong HH, Li YX, Stahl GL, Thurman JM. Enhanced susceptibility to acute pneumococcal otitis media in mice deficient in complement C1qa, factor B, and factor B/C2. Infect Immun. 2010; 78:976-83.

10. Tong HH, Long JP, Li D, DeMaria TF. Alteration of gene expression in human middle ear epithelial cells induced by influenza A virus and its implication for the pathogenesis of otitis media. Microb Pathog. 2004; 37:193-204. 
11. Thorgersen EB, Barratt-Due A, Haugaa H, Harboe M, Pischke SE, Nilsson PH, et al. The Role of Complement in Liver Injury, Regeneration, and Transplantation. Hepatology. 2019; 70:725-36.

12. He S, Atkinson C, Qiao F, Cianflone K, Chen X, Tomlinson S. A complement-dependent balance between hepatic ischemia/reperfusion injury and liver regeneration in mice. J Clin Invest. 2009; 119:2304-16.

13. He S, Atkinson C, Evans Z, Ellett JD, Southwood M, Elvington A, et al. A role for complement in the enhanced susceptibility of steatotic livers to ischemia and reperfusion injury. J Immunol. 2009; 183:4764-72.

14. Singhal R, Ganey PE, Roth RA. Complement activation in acetaminophen-induced liver injury in mice. J Pharmacol Exp Ther. 2012; 341:377-85.

15. Zhang GL, Zhang T, Ye YN, Liu J, Zhang XH, Xie C, et al. Complement Factor 3 Could Be an Independent Risk Factor for Mortality in Patients with HBV Related Acute-on-Chronic Liver Failure. Biomed Res Int. 2016; 2016:3524842.

16. Rensen SS, Slaats Y, Driessen A, Peutz-Kootstra CJ, Nijhuis J, Steffensen R, et al. Activation of the complement system in human nonalcoholic fatty liver disease. Hepatology. 2009; 50:1809-17.

17. Wiesner R, Edwards E, Freeman R, Harper A, Kim R, Kamath P, et al. Model for end-stage liver disease (MELD) and allocation of donor livers. Gastroenterology. 2003; 124:91-6.

18. Li N, Huang C, Yu KK, Lu Q, Shi GF, Zheng JM. Validation of prognostic scores to predict short-term mortality in patients with HBV-related acute-on-chronic liver failure: The CLIF-C OF is superior to MELD, CLIF SOFA, and CLIF-C ACLF. Medicine (Baltimore). 2017; 96:e6802.

19. Terrault NA, Lok ASF, McMahon BJ, Chang KM, Hwang JP, Jonas MM, et al. Update on prevention, diagnosis, and treatment of chronic hepatitis B: AASLD 2018 hepatitis B guidance. Hepatology. 2018; 67:1560-99.

20. Jalan R, Saliba F, Pavesi M, Amoros A, Moreau R, Ginès P, et al. Development and validation of a prognostic score to predict mortality in patients with acute-on-chronic liver failure. J Hepatol. 2014; 61:1038-47.

21. Laursen TL, Sandahl TD, Støy S, Schiødt FV, Lee WM, Vilstrup H, et al. Circulating mannan-binding lectin, M-, L-, H-ficolin and collectin-liver-1 levels in patients with acute liver failure. Liver Int. 2015; 35:756-63.

22. Schraufstatter IU, Khaldoyanidi SK, DiScipio RG. Complement activation in the context of stem cells and tissue repair. World J Stem Cells. 2015; 7:1090-108.

23. Markiewski MM, Mastellos D, Tudoran R, DeAngelis RA, Strey CW, Franchini S, et al. C3a and C3b activation products of the third component of complement (C3) are critical for normal liver recovery after toxic injury. J Immunol. 2004; 173:747-54.

24. Mastellos D, Papadimitriou JC, Franchini S, Tsonis PA, Lambris JD. A novel role of complement: mice deficient in the fifth component of complement (C5) exhibit impaired liver regeneration. $J$ Immunol. $2001 ; 166: 2479-86$. 
25. Markiewski MM, DeAngelis RA, Strey CW, Foukas PG, Gerard C, Gerard N, et al. The regulation of liver cell survival by complement. J Immunol. 2009; 182:5412-8.

26. Strey CW, Markiewski M, Mastellos D, Tudoran R, Spruce LA, Greenbaum LE, et al. The proinflammatory mediators C3a and C5a are essential for liver regeneration. J Exp Med. 2003; 198:913-23.

27. Daveau M, Benard M, Scotte M, Schouft MT, Hiron M, Francois A, et al. Expression of a functional $\mathrm{C} 5$ a receptor in regenerating hepatocytes and its involvement in a proliferative signaling pathway in rat. J Immunol. 2004; 173:3418-24.

\section{Tables}

Table 1. Baseline characteristics of the HBV-ACLF, CHB group and health control groups.

\begin{tabular}{|c|c|c|c|c|}
\hline Characteristics & $\begin{array}{l}\text { Control } \\
\text { group }\end{array}$ & CHB group & ACLF group & P Value \\
\hline Patients, no. & 9 & 25 & 27 & \\
\hline Age, y(median, range) & $37(29-50)$ & $31(15-55)$ & $42(24-59)$ & 0.1182 \\
\hline Gender, $\mathrm{M} / \mathrm{F}$ & $5 / 4$ & $16 / 9$ & $23 / 4$ & 0.1510 \\
\hline ALT (IU/L) (median, range) & $19(15-38)$ & $25(13-106)$ & $230(17-$ & $<0.0001$ \\
\hline Bilirubin $(\mu \mathrm{mol} / \mathrm{L})$ (median, & 10.5 & $12.2(5.2-$ & $314.9(20-$ & $<0.0001$ \\
\hline Albumin (g/L) (median, & ND & $46(33-49)$ & $32(27-42)$ & $<0.0001^{*}$ \\
\hline Creatinine $(\mu \mathrm{mol} / \mathrm{L})$ & $78(47-93)$ & $73(36-80)$ & $67(21-646)$ & 0.8256 \\
\hline $\begin{array}{l}\text { INR } \\
\text { INRan, range) }\end{array}$ & ND & $\begin{array}{l}1.03(0.90- \\
1.10)\end{array}$ & $\begin{array}{l}1.90(1.07- \\
4.93)\end{array}$ & $<0.0001 *$ \\
\hline $\begin{array}{l}\text { AFP (ng/ml) (median, } \\
\text { range) }\end{array}$ & ND & $2.02(1.07-$ & $\begin{array}{l}59.98(3.85- \\
9598)\end{array}$ & $<0.0001^{*}$ \\
\hline HBeAg positive, n(\%) & 0 & $13(52 \%)$ & $17(63 \%)$ & $0.9506^{*}$ \\
\hline $\begin{array}{l}\text { HBV DNA }\left(\log _{10} \mathrm{IU} / \mathrm{ml}\right) \\
\text { (median, range) }\end{array}$ & ND & $\begin{array}{l}7.453(2.699- \\
8.656)\end{array}$ & $\begin{array}{l}4.189(2.699- \\
7.477)\end{array}$ & $0.0178 *$ \\
\hline MELDs (median, range) & ND & $2(2-6)$ & $23(10-43)$ & $0.0005 *$ \\
\hline CLIF-C OFs (median, range) & ND & $6(6-6)$ & $8(6-12)$ & $\begin{array}{l}\text { Cannot be } \\
\text { calculated }\end{array}$ \\
\hline
\end{tabular}

Continuous variables were presented as median and the range was shown in brackets. ND: not detected. * If the test was not detected, the other two group was compared.

Table 2. Plasma levels of complement components in the HBV-ACLF, CHB group and health control groups 


\begin{tabular}{lllll}
\hline Characteristics & Control group & CHB group & ACLF group & $\begin{array}{l}\text { Value } \\
\end{array}$ \\
\hline C1q $(\mathrm{ng} / \mathrm{ml})$ & 177001(145157- & $114640(34586-$ & $50509(16011-$ & $<$ \\
MBL $(\mathrm{ng} / \mathrm{ml})$ & 374153) & $198231)$ & $113313)$ & 0.0001 \\
FB $(\mathrm{ng} / \mathrm{ml})$ & $236.5(147.8-303.8)$ & $214.3(176.0-270.6)$ & $222.4(141.8-$ & 0.7615 \\
C3 $(\mu \mathrm{g} / \mathrm{ml})$ & $15653(12550-$ & $8916(3598-16893)$ & $6568(1188-$ & $<$ \\
C3a $(\mathrm{ng} / \mathrm{ml})$ & $16220)$ & $1008(894-1654)$ & $852(714-1740)$ & $<$ \\
C4 $(\mu \mathrm{g} / \mathrm{ml})$ & $1530(498-1843)$ & $1487(76-2006)$ & $1074(49-1554)$ & 0.0001 \\
C4a $(\mathrm{ng} / \mathrm{ml})$ & $2398(1086-3013)$ & $2304(1575-3007)$ & $1811(417-2757)$ & 0.00034 \\
sC5b-9 $(\mathrm{ng} / \mathrm{ml})$ & $784(445-2626)$ & $396(121-2288)$ & $130(79-1772)$ & 0.0115 \\
\hline
\end{tabular}

Continuous variables were presented as median and the range was shown in brackets.

\section{Figures}
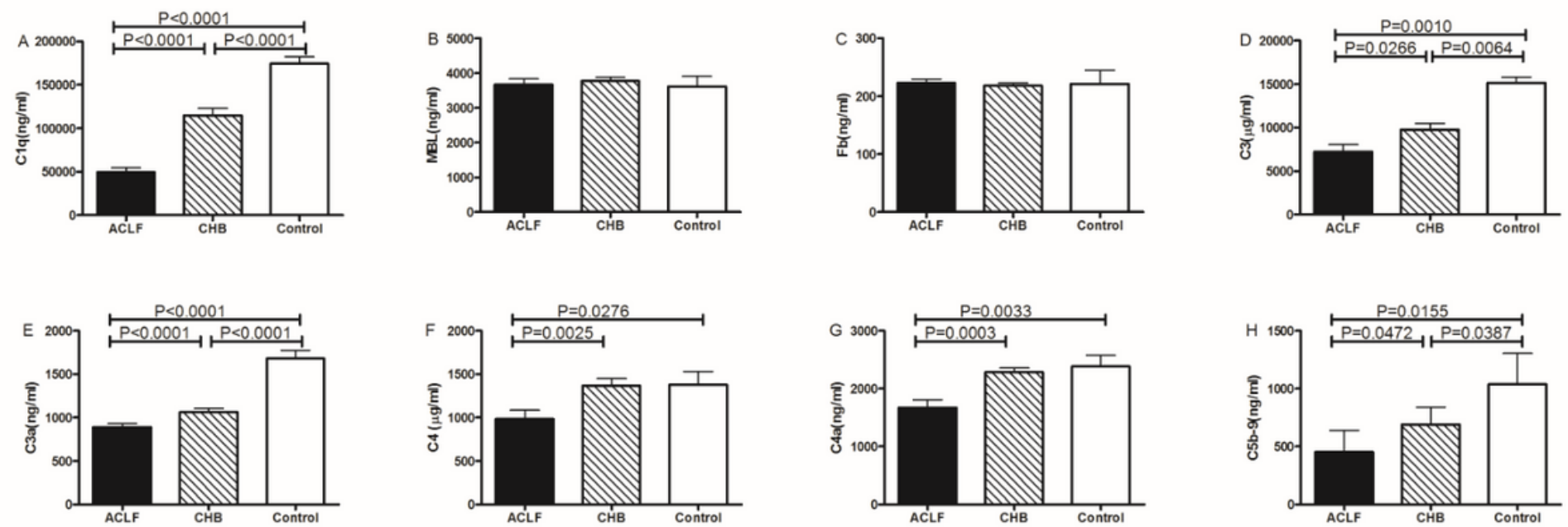

Figure 1

Complement in ACLF group, $\mathrm{CHB}$ group and control group. The concentrations of $\mathrm{C} 1 \mathrm{q}$ were significantly lower in HBV-ACLF group than that in CHB group or in control group(Fig. 1A). However, There were no statistical significances of the plasma concentrations of MBL and FB in all groups(Fig. 1B-C). Complement $\mathrm{C} 3, \mathrm{C} 3 \mathrm{a}, \mathrm{C} 4, \mathrm{C} 4 \mathrm{a}$ and its degradation product $\mathrm{C} 5 \mathrm{~b}-9$ were significantly higher in the control group than that in the HBV-ACLF group(Fig. 1D-H). 

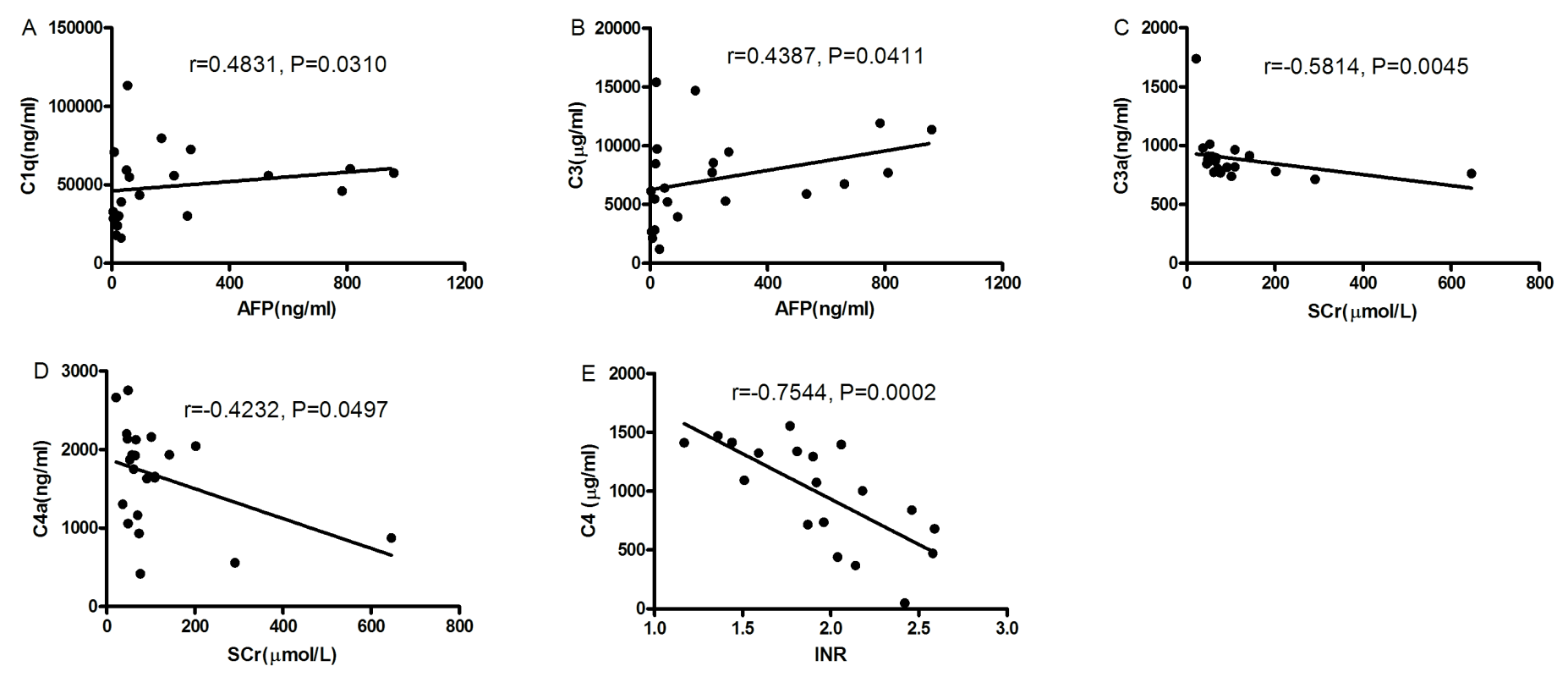

\section{Figure 2}

Relationship between complement components and clinical parameter. C1q and C3 were observed to be positively correlated with AFP $(\mathrm{P}<0.05)$ (Fig. 2A-B). C3a and C4a were observed to be negatively correlated with creatinine $(P<0.05)$ (Fig. $2 C-D)$. C4 were observed to be negatively correlated with INR $(P$ $<0.05)$ (Fig. 2E).
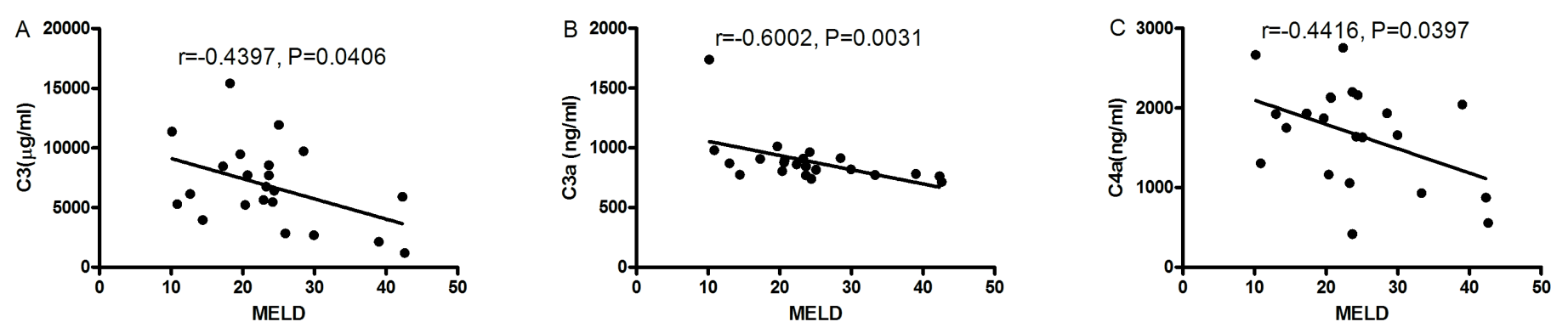

Figure 3

Correlation of $\mathrm{C} 3, \mathrm{C} 3 \mathrm{a}$ and $\mathrm{C} 4 \mathrm{a}$ with prognostic scoring systems. $\mathrm{C} 3, \mathrm{C} 3 \mathrm{a}$ and $\mathrm{C} 4 \mathrm{a}$ were observed to be negatively correlated with MELDs $(P<0.05)($ Fig. $3 A-C)$. 


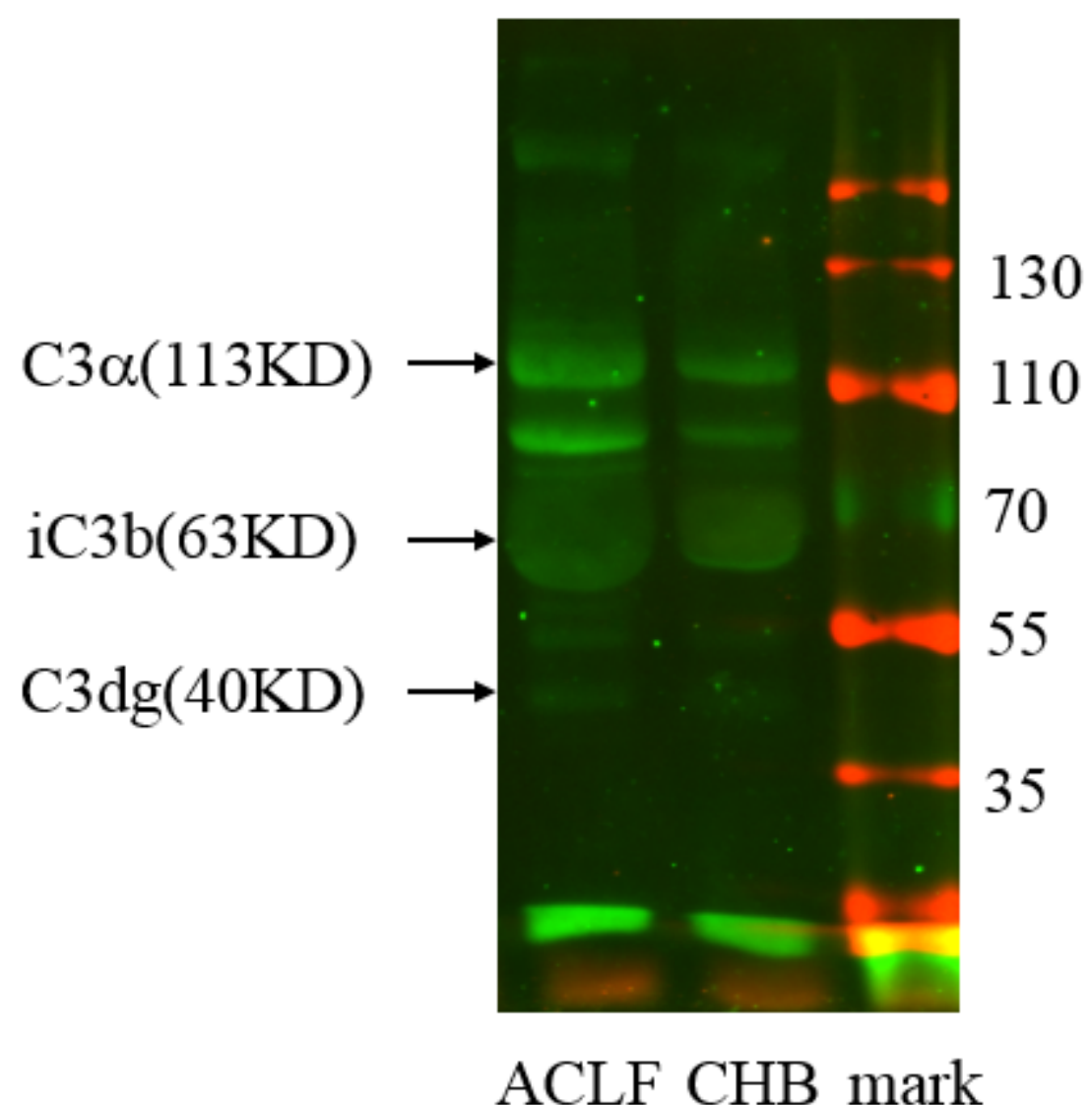

\section{Figure 4}

C3 cleavage fragments were analyzed by western blot. The immunoreactive bands corresponding to C3a, iC3b and C3dg are denoted. Under the same C3 concentration, the C3 breakdown products iC3b was more in HBV-ACLF group than the one in CHB group. Prominent iC3b band was found in plasma samples in HBV-ACLF patients. 